CONCISE REPORT

\title{
Imaging of tophaceous gout: computed tomography provides specific images compared with magnetic resonance imaging and ultrasonography
}

\author{
J C Gerster, M Landry, L Dufresne, J Y Meuwly
}

Ann Rheum Dis 2002;61:52-54

Objective: To determine the usefulness of computed tomography (CT), magnetic resonance imaging (MRI), and Doppler ultrasonography (US) in providing specific images of gouty tophi.

Methods: Four male patients with chronic gout with tophi affecting the knee joints (three cases) or the olecranon processes of the elbows (one case) were assessed. Crystallographic analyses of the synovial fluid or tissue aspirates of the areas of interest were made with polarising light microscopy, alizarin red staining, and $x$ ray diffraction. CT was performed with a GE scanner, MR imaging was obtained with a $1.5 \mathrm{~T}$ Magneton (Siemens), and ultrasonography with colour Doppler was carried out by standard technique.

Results: Crystallographic analyses showed monosodium urate (MSU) crystals in the specimens of the four patients; hydroxyapatite and calcium pyrophosphate dihydrate (CPPD) crystals were not found. A diffuse soft tissue thickening was seen on plain radiographs but no calcifications or ossifications of the tophi. CT disclosed lesions containing round and oval opacities, with a mean density of about 160 Hounsfield units (HU). With MRI, lesions were of low to intermediate signal intensity on $T_{1}$ and $T_{2}$ weighting. After contrast injection in two cases, enhancement of the tophus was seen in one. Colour Doppler US showed the tophi to be hypoechogenic with peripheral increase of the blood flow in three cases.

Conclusion: The MR and colour Doppler US images showed the tophi as masses surrounded by a hypervascular area, which cannot be considered as specific for gout. But on CT images, masses of about $160 \mathrm{HU}$ density were clearly seen, which correspond to MSU crystal deposits.

n previous studies we have shown that MSU crystal deposits in chronic gout may be identified by CT in the knee joints, ${ }^{1}$ in the tendons, ${ }^{2}$ and in subcutaneous tissues. ${ }^{3}$ They appear as round and oval opacities having a CT attenuation in the range of 150-200 HU. This study aimed at comparing CT with magnetic resonance MR imaging and

\section{PATIENTS AND METHODS}

Four men with chronic tophaceous gout were examined. Their mean age was 56.3 years and the mean duration of gout 18 years. Associated disorders were hypertension and type II diabetes (patient 1), obesity and gonarthrosis (patient 2), allergy to allopurinol (patient 3), and hypertension (patient 4). Imaging of the left olecranon was carried out in patient 1 , the left knee in patient 2, and the prepatellar bursa in patients 3 and 4 .

\section{Identification of crystals}

Needle aspiration of synovial fluid (patients 2,3,4) or tophaceous material (patient 1) was made in the area which was imaged. The aspirates were examined with polarising light microscopy and a first order red filter, then they were coloured with alizarin red stain, according to the technique of Paul et al for detection of apatite. ${ }^{4}$ The samples were analysed with powder diffraction $x$ ray by standard methods. ${ }^{5}$

\section{Imaging technique}

Conventional $x$ ray examinations were made in all the cases. CT examination was performed with a 9800 high speed advantage scanner (GE), MR imaging was obtained with a 1.5 $\mathrm{T}$ magneton (Siemens), and ultrasonography with colour Doppler was carried out with a high frequency pulse (Acuson $128 \mathrm{XP} / 10$ or an ATL HDI 5000).

\section{RESULTS}

Identification of crystals

Polarising light microscopy disclosed MSU crystals; CPPD crystals were not seen. Alizarin red staining did not show calcium containing compounds in any of the patients. $x$ Ray powder diffraction analyses identified MSU crystals in the samples of all four patients. Apatite crystals were not found.

\section{Results of imaging techniques}

Plain radiographs showed soft tissue thickening; there were no calcifications or ossifications within the tophi. Bone erosions in contact with tophi were seen in patients 2, 3, and 4. CT images disclosed round and oval opacities in the tophi, 2-6 $\mathrm{mm}$ in diameter, with a mean density of about $160 \mathrm{HU}$ (figs 1A and 2A). On MR imaging, the tophi were of intermediate intensity on $\mathrm{T}_{1}$ weighting and of intermediate to low intensity on $\mathrm{T}_{2}$ weighting (figs $\mathrm{IB}$ and $2 \mathrm{~B}$ ). A gadolinium injection was made in patients 1 and 2 ; there was no enhancement in case 1 and in case 2 the signal was enhanced around the tophus (fig 1B). On gray scale ultrasound images, the tophi were hypoechogenic with bright spots in the periphery and some shadowing (figs $\mathrm{IC}$ and $2 \mathrm{C}$ ). With colour Doppler, there was no obvious vascularity in case 1 . However in cases 2, 3, and 4 increased vascularity was seen around the tophi (figs $1 \mathrm{C}$ and 2C).

\section{DISCUSSION}

An imaging technique which could specifically demonstrate MSU deposits within tissues would be very helpful, especially in patients presenting with a tumour of soft tissues of

Abbreviations: $C T$, computed tomography; $C P P D$, calcium pyrophosphate dihydrate; HU, Hounsfield units; MRI, magnetic resonance imaging; MSU, monosodium urate; US, ultrasound 
unknown cause ${ }^{6}$ or in patients with subcutaneous noduleswhich might be related either to gout or to rheumatoid arthritis. ${ }^{7}$ As we have shown in previous studies, MSU deposits within a tophus can be clearly defined with CT. The opacities have a CT attenuation of about $160 \mathrm{HU}$, which is clearly inferior to that of deposits of calcium containing crystals, which have an attenuation of about 450 HU. Apatite crystals can be seen in old tophi. This is shown by plain $x$ rays, which sometimes disclose calcifications or ossifications in the centre of some tophi ${ }^{7}$; in some cases anatomopathological studies have also disclosed calcifications in necrotic centres of tophi. ${ }^{9}$

However, there were no radiological or crystallographic data supporting the idea that apatite crystals might have been present in our cases. Unlike the results of Chen et al, ${ }_{1}^{10}$ our data do not sustain the hypothesis that apatite crystals mixed with MSU crystals may explain the opacities seen on CT images. Moreover, pure MSU crystal deposits have been proved to have the same CT attenuation in vitro as in tissues. $^{1}$

On ultrasonographic images, the tophi appeared as hypoechogenic structures of high attenuation with shadowing and hyperechogenic surrounding, images which cannot be clearly differentiated from rheumatoid nodules. ${ }^{11}$ MR imaging showed the tophi as structures of intermediate to low signal intensity, as already reported in previous papers. ${ }^{6}{ }^{10} 12$ These findings cannot be considered specific to gout. ${ }^{12}$ The post-gadolinium injected images showed a peripheral enhancement of the tophus (fig 1B), which is a sign of hypervascularity in patient 2 (fig $1 \mathrm{~B}$ ) but not in patient 1; likewise images with colour Doppler showed hypervascularity in patient 2 as well as in patients 3 and 4. It can be asked whether hypervascularity as demonstrated on injected MR and colour Doppler images is a sign of inflammatory reaction. Indeed, hypervascularity could favour bone erosions and destruction in close contact with the tophi, as in the three cases in which images of hypervascularity were seen; bone erosions were absent in the patient without signs of hypervascularity.

\section{ACKNOWLEDGMENT}

The authors thank L Calame, chief technician, for synovial fluid analyses, and P Thélin, of the University Institute of Mineralogy of Lausanne for supervising $x$ ray diffraction analyses.
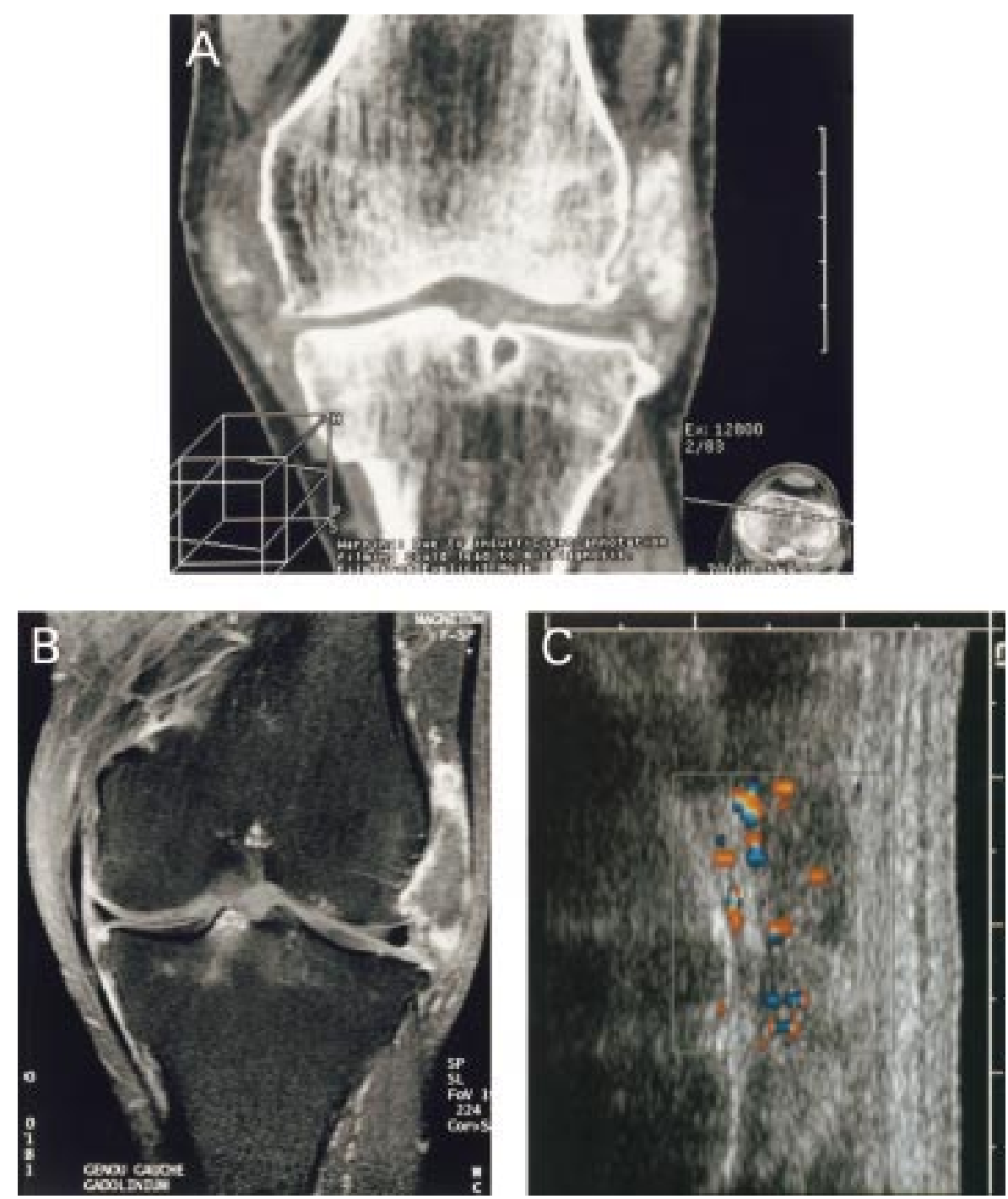

Figure 1 (A) Patient 2, left knee, lateral tophus. Two dimensional reconstruction coronal CT: lateral soft tissue thickening containing nodular opacities of 150-200 HU in density (a smaller medial tophus lesion is seen). (B) Coronal T, MRI with fat saturation after injection of gadolinium. The lateral tophus is intermediate in signal intensity. The periphery of the tophus is stained with contrast. (C) Colour Doppler view of the lateral aspect of the knee: the tophus is hypoechogenic with an hyperechogenic rim. Vessels are seen all around the tophus (coloured spots). 

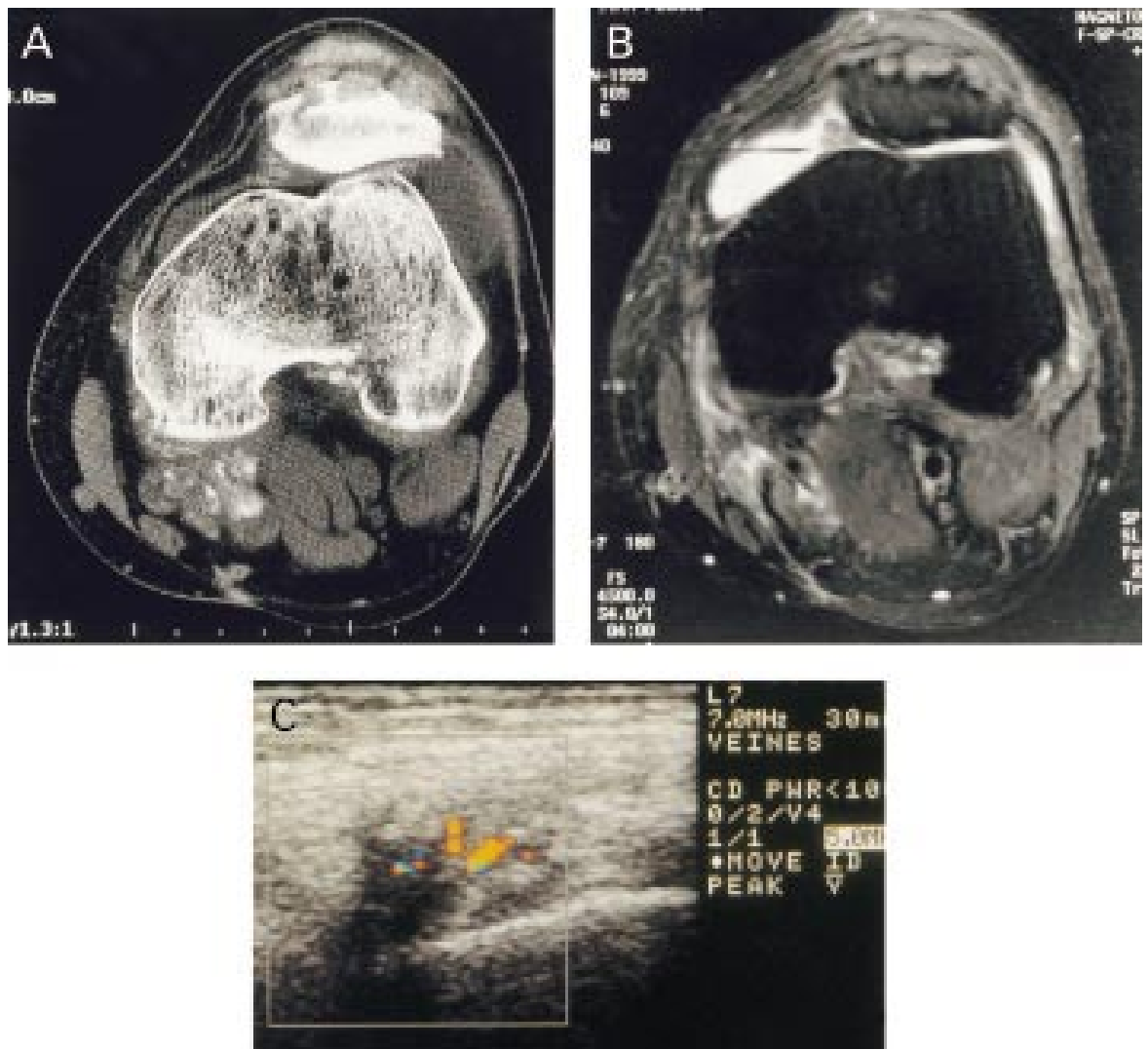

Figure 2 (A) Patient 3, left knee, prepatellar tophus. CT showing prepatellar soft tissue thickening and small nodular opacities of 150-200 $\mathrm{HU}$ in density. There are erosions with overhanging edges of the patella. Nodular opacities are also seen in the posterior part of the knee. (B) Transverse $\mathrm{T}_{2}$ MRI with fat saturation. The soft tissue is intermediate in signal intensity. The nodular lesions are clearly seen. There is also joint fluid. (C) Colour Doppler transverse view of the medial aspect of the knee. The tophus appears as a hypoechogenic nodule surrounded by a hyperechogenic rim. Some vessels are visible within and around the nodule.

\section{Authors' affiliations}

J C Gerster Department of Rheumatology, University Hospital (CHUV), Lausanne, Switzerland

M Landry, J Y Meuwly Department of Radiodiagnostics, CHUV Lausanne, Switzerland

L Dufresne Institute of Mineralogy, University of Lausanne, Switzerland.

Correspondence to: Professor J C Gerster, Service de Rhumatologie, CHUV, 1011 Lausanne, Switzerland;

Jean-Charles.Gerster@chuv.hospvd.ch

Accepted 14 May 2001

\section{REFERENCES}

1 Gerster JC, Landry M, Duvoisin B, Rappoport G. Computed tomography of the knee joint as an indicator of intraarticular tophi in gout. Arthritis Rheum 1996;39:1406-9.

2 Gerster JC, Landry M, Rappoport G, Rivier G, Duvoisin B, Schnyder P.

Enthesopathy and tendinopathy in gout: computed tomographic assessment. Ann Rheum Dis 1996;55:921-3.
3 Gerster JC, Landry M, Rivier G. Computed tomographic imaging of subcutaneous gouty tophi. Clin Rheumatol 1998;17:62-4.

4 Paul H, Reginato AJ, Schumacher HR. Alizarin red S staining as a screening test to detect calcium compounds in synovial fluid. Arthritis Rheum 1983;26:191-200.

5 Bunn CW. Chemical crystallography. 2nd ed. Oxford: Clarendon Press, 1961.

6 Chaoui A, Garcia J, Kurt AM. Gouty tophus simulating soft tissue tumor in a heart transplant recipient. Skeletal Radiol 1997;26:626-8.

7 Talbott JH, Altman RD, Yü TF. Gouty arthritis masquerading as rheumatoid arthritis or vice versa. Semin Arthritis Rheum 1978;8:77-114.

8 Dieppe P, Calvert P. Crystals and joint disease. London, New York: Chapman and Hall, 1983.

9 Fassbender HG. Pathology of rheumatic diseases. Berlin, Heidelberg New York: Springer, 1975.

10 Chen CKH, Ren Yeh L, Pan HB, Yang CF, Lu YC, Resnick D. Intra-articular gouty tophi of the knee: CT and MR imaging in 12 patients. Skeletal Radiol 1999;28:75-80.

11 Tiliakos N, Morales AR, Wilson CH. Use of ultrasound in identifying tophaceous versus rheumatoid nodules. Arthritis Rheum 1982;25:478-9.

12 Yü JS, Chung C, Recht M, Dailiana T, Jurdi R. MR imaging of tophaceous gout. AJR 1997; 168:523-7. 